\title{
Discovering perfect squares and building square roots
}

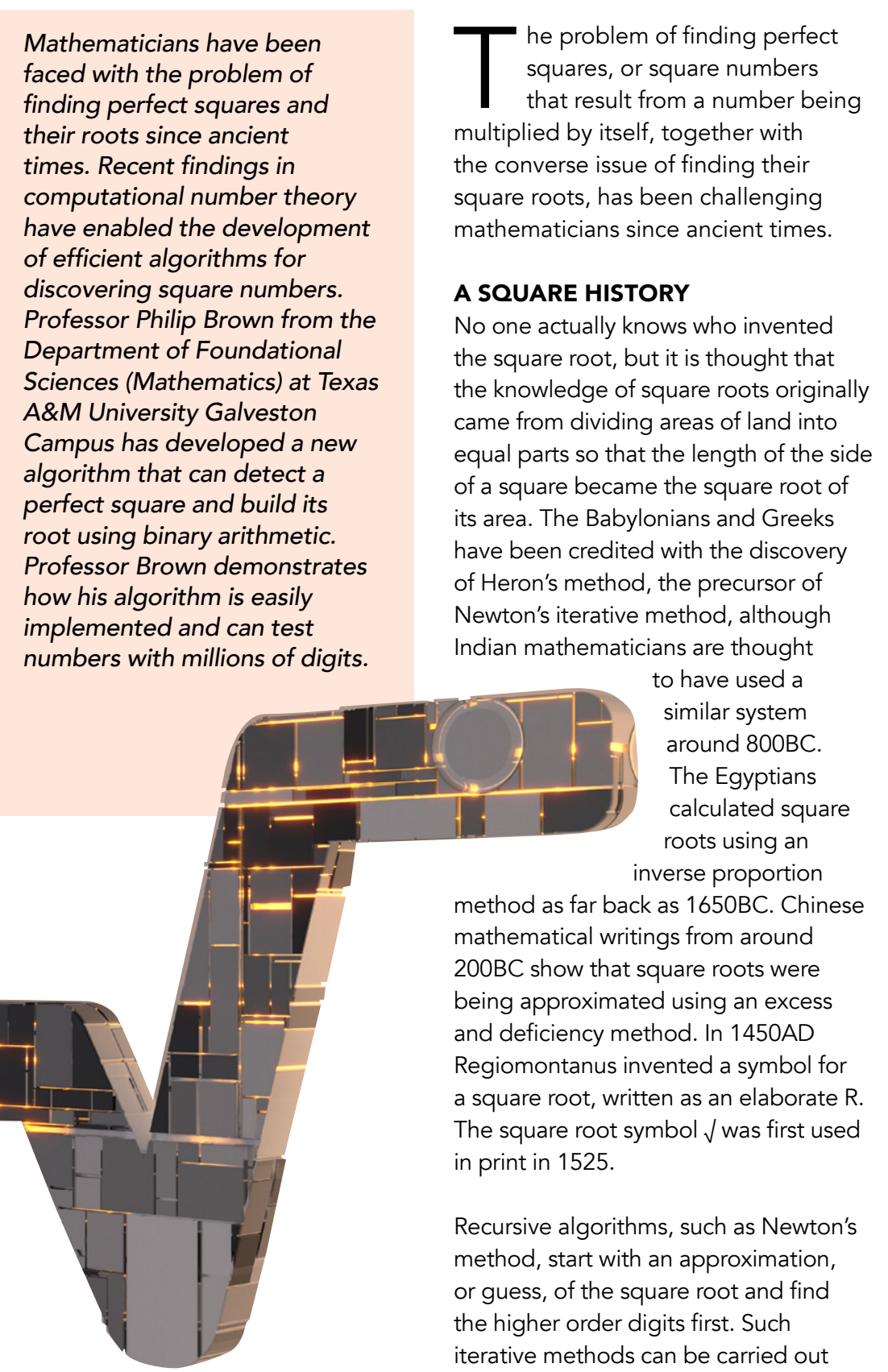

on a computer using floating point tithmetic, but they are usually difficult and computational difficulty can arise with the division operation. More recently computational number theory the area of number theory concerned with finding and implementing efficient computer algorithms, has enabled. the development of algorithms involving sieve methods to decide whether or not a positive integer is a perfect power.

Prof Philip Brown from the Department of Foundational Sciences (Mathematics) at Texas A\&M University Galveston Campus has developed a new algorithm for discovering square numbers. Combining elementary number theory and algorithmic number theory, this novel algorithm multiplication, so the potentially problematic division operation is not required. The algorithm is easily implemented and can test numbers with millions of digits. Furthermore this new algorithm tests with certainty whether or not a number is a perfect square, in contrast with other methods that can only test to a high degree of probability.

Konnor Chappell (a student at Texas A\&M University at Galveston) helped Professor Brown write the Python code

\section{REVEALING PROPERTIES}

OF PERFECT SQUARES

some properties of perfect squat

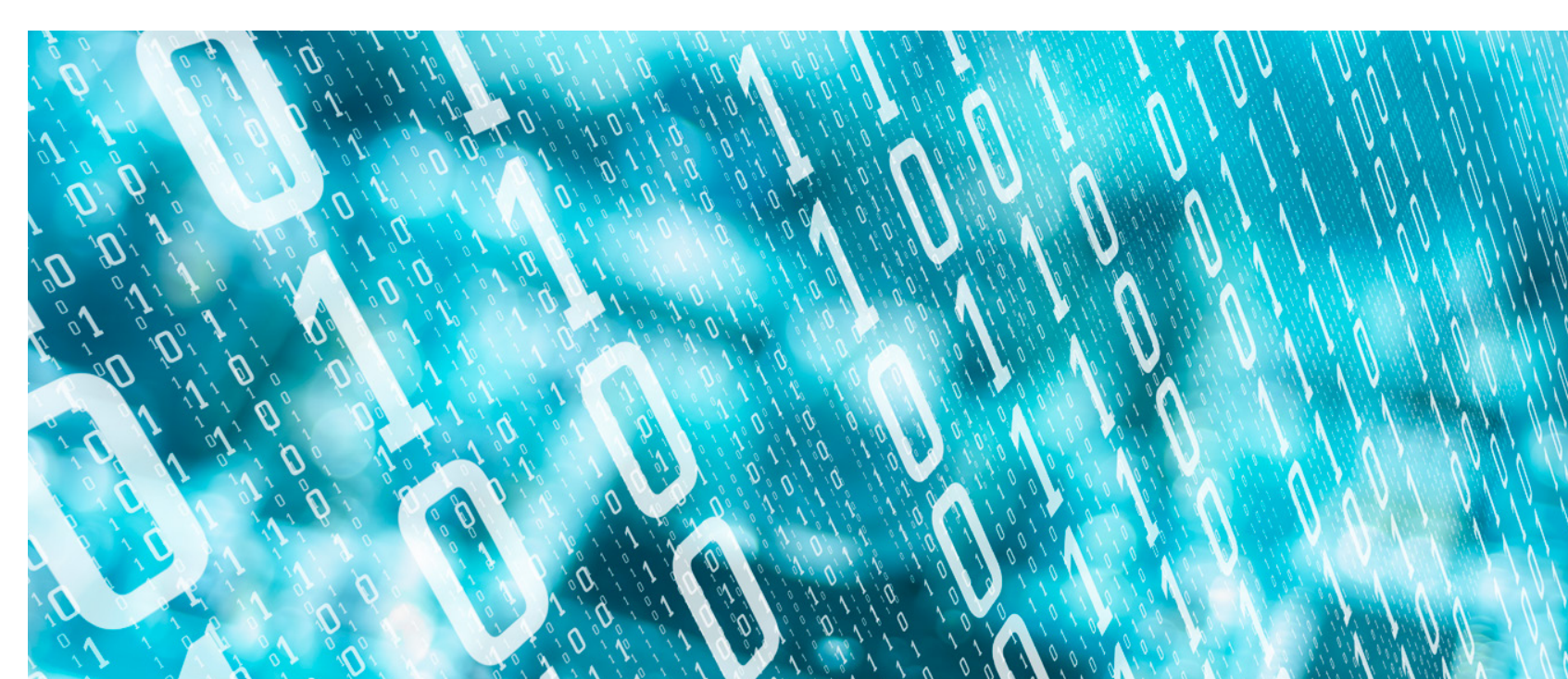

are revealed if the numbers are expressed in a base that is a power of 2, such as in the binary, octal and underpins how his algorithm can identify a perfect square and build tis square root, starting with the low order digits and working through to the high order digits.

Because the algorithm builds square roots starting with the lower order digits, it is easier to comprehend if we read or label the digits from right to left.

Prof Brown demonstrates how the algorithm starts by converting the input number N from base 10 to base 2. If $N$ is an even number, then its base 2 expression begins (on the right hand side) with a string of binary digits th are all equal to 0 , e.g. the decimal 100 and 10100 in base 2, respectively. When the input number, $N$, is an even number the initial string of zeros of the binary representation of the number is truncated, leaving the resulting odd number to be tested. If this odd number is a square, then $N$ is either a square or twice a square. This means that the algorithm only needs to continue when $N$ is odd. So given an odd integer $\mathrm{N}$ in octal format the algorithm will determine if $N$ is a perfect square and compute the squar oot if required.

OBSERVATIONS WITH BASE 8 ase 8 is a convenient number system to demonstrate Prof Brown's

Multiplication by 4 in base 8 , e.g. $1 \times 4=4,2 \times 4=10$, always results in a side. Cons a squares expressed in base 8 begin (reading from right to left) with a 0 or 4. Prof Brown also shows that all odd perfect squares expressed in base 8 begin with a 1 .

BASE $2^{S}$

Moreover, Prof Brown demonstrates that for bases that are higher powers of 2 , denoted $2^{s}$, if the first base $2^{s}$ dig of some number $N$ is not congruent
(modulo $2^{5}$ ) to the square of an odd 政 a square number.

TIME COMPLEXIT

The time complexity of an algorithm antifies the amount of time that an gorithm takes to run as a function of the length of the input. Prof Brown's algorithm tests whether a positive is a square number, and/ or computes the square root of $N$ has is themplexity of $\mathrm{O}\left(\left(\log ^{2} N\right) / \mathrm{s}\right)$ where sis the power of the chosen base - for base 16. This 'big O' 8 and $s=4$ tor that the algorithm's performance is

Professor Brown has developed a theoretical basis for this algorithm that provides new insight into the properties of square numbers.
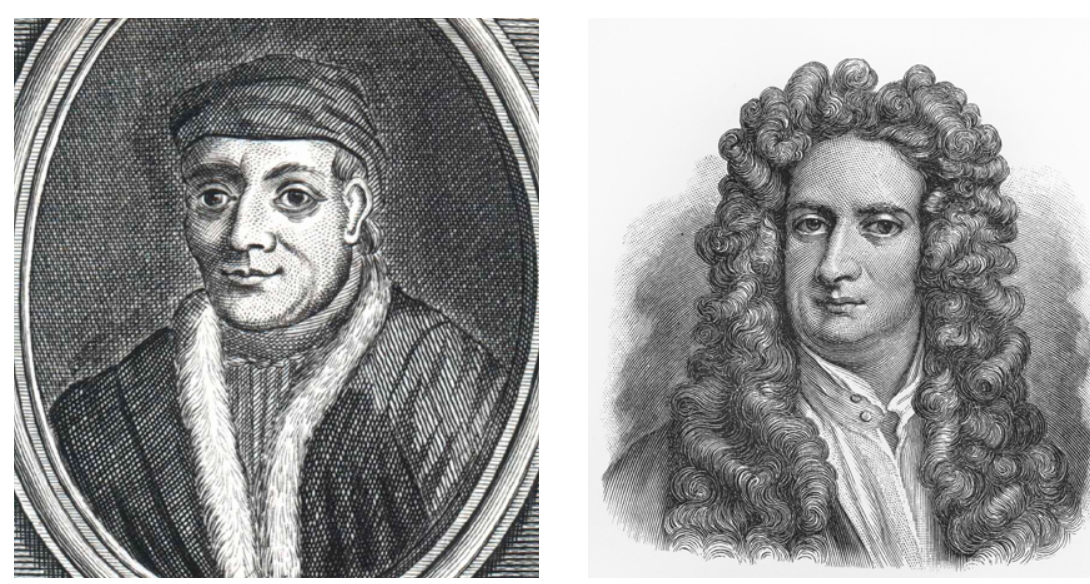

Regiomontanus (left) is considered the inventor of the square root symbol. Isaac Newton (right 

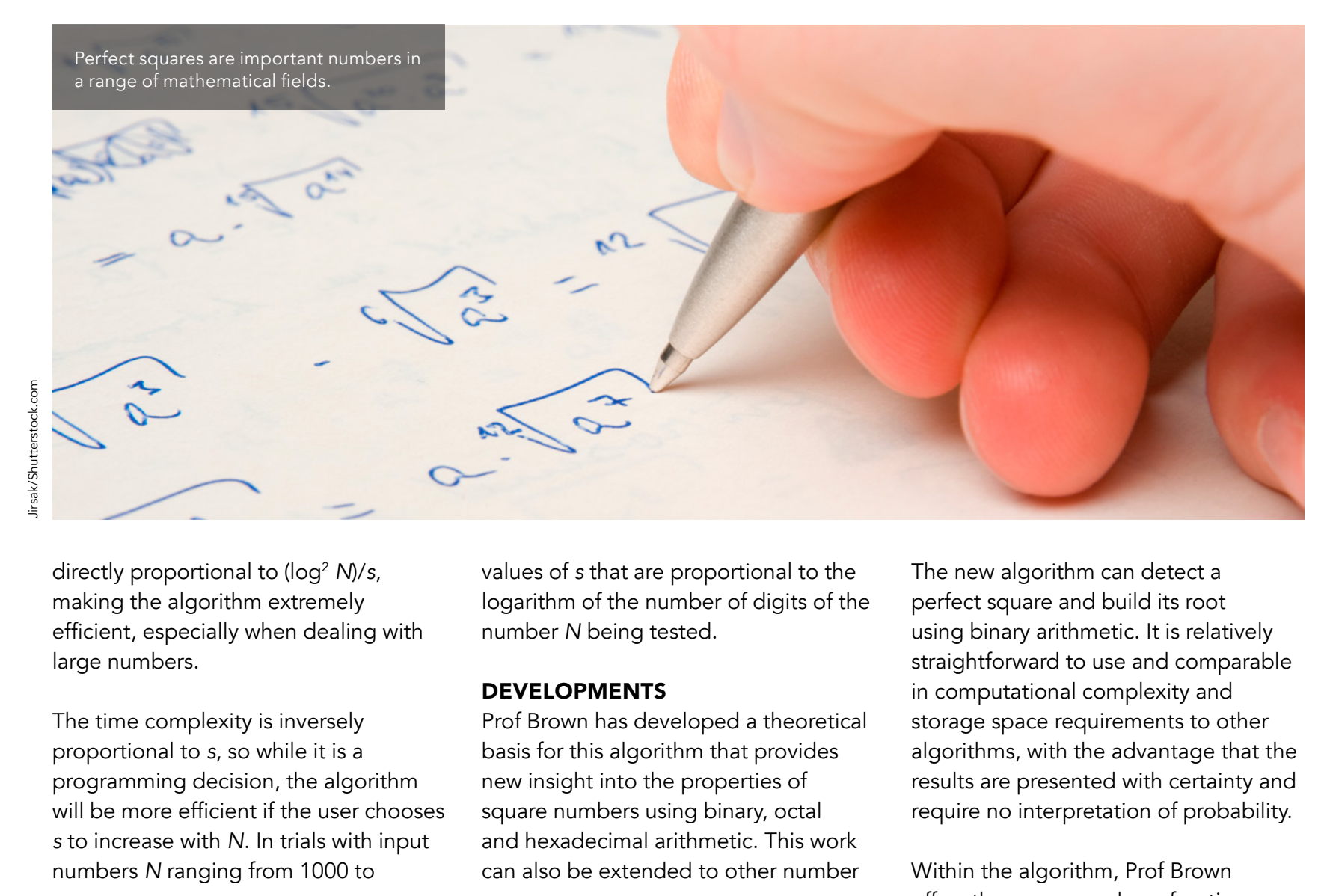

values of $s$ that are proportional to the logarithm of the number of digits of the number $N$ being tested.

\section{DEVELOPMENTS}

Prof Brown has developed a theoretica new insight into the properties of

square numbers using binary, octal and hexadecimal arithmetic. This work can also be extended to other number

The new algorithm can detect a perfect square and build its root using binary arithmetic.

512000 digits, Prof Brown has observed systems with bases that are even large that there appears to be optimal powers of 2

Binary System Table
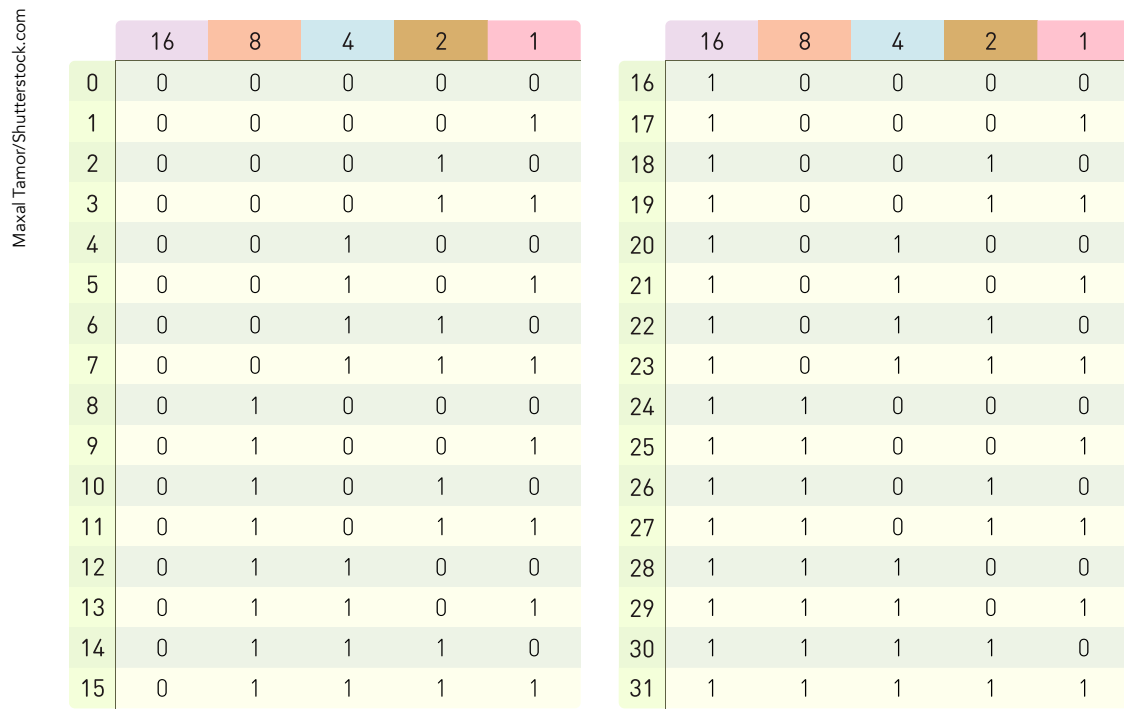

The new algorithm can detect a perfect square and build its root using binary arithmetic. It is relatively
straightforward to use and comparable straightforward to use and comp ra storage space requirements to other algorithms, with the advantage the results are presented with certainty and equire no interpretation of probability.

Within the algorithm, Prof Brown offers the user a number of options to tailor the algorithm to suit their individual requirements. For instance, the programmer can opt to solve for multiplicative inverses by using deploying the extended Euclidean algorithm. They also have the choice of implementing the algorithm in base 8 or any other base that is a higher power 2 , which may depend on the

\section{ONGOING AND FUTURE WORK} The algorithm runs efficiently using a power of 2. Currently, there is no other bases such as base 10. Prof Brown is busy developing a recursive implementation of the binary algorithm that uses a smaller value of $s$ at each level of recursion, beginning with This algorithm has an improved time complexity of $O\left(\log N \log ^{2} \log N\right)$ and it of digits using a desktop computer in less than one hour. He is also considering whether the algorithm can greater than 2, such as perfect cubes. pre-computed selection matrices or straightforward way of employing a value of $s$ proportional to $\log N$. is possible to test numbers with billion

\section{Behind the Research}

\section{Professor Philip Brown}

E: brownp@tamug.edu T: $+14095993579 \quad$ F: +14097414388

W: http://www.tamug.edu/fsci/faculty-bios/BrownP.htm/

Research Objectives

Professor Brown has developed a new algorithm for discovering square numbers.

\section{Detail}

Philip Brown

Galveston

Texas 77550

\section{Bio}

After completing his undergraduate studies in Johannesburg, South Africa, Prof Brown enrolled in the mathematics graduate program at Texas A\&M University. His PhD dissertation and subsequent functions of has been primarily in the study of a textbook titled Foundations of Mathematics.

\section{Collaborators}

Konnor Chappell (a student at Texas A\&M University at Galveston) helped write the Python code in order to implement the algorithm.

\section{References}

Brown, P.R. (2019). Detecting square numbers. Quaestiones Mathematicae, [online]. Available at: https://doi.org/10.2989/16073606.2019.1678530

Bernstein, D.J. (1998). Detecting perfect powers in essentially linear time. Mathematics of Computation

Bach, E. and Sorenson, J. (1993). Sieve Algorithms for Perfect Power Testing. Algorithmica, 9, 13-328.

\section{Personal Response}

What initially inspired you to develop a new II I enjoy working on fundamental problems in
mathematics and science. During my career I have published research work relating to certain fundamental constars (including tha fine structure constanber $\pi$ ) and interested in the problem of detecting square numbers when my mother, Ria Brown, who was a high school mathematics teacher, pointed out to me a pattern in the digits of perfect squares.

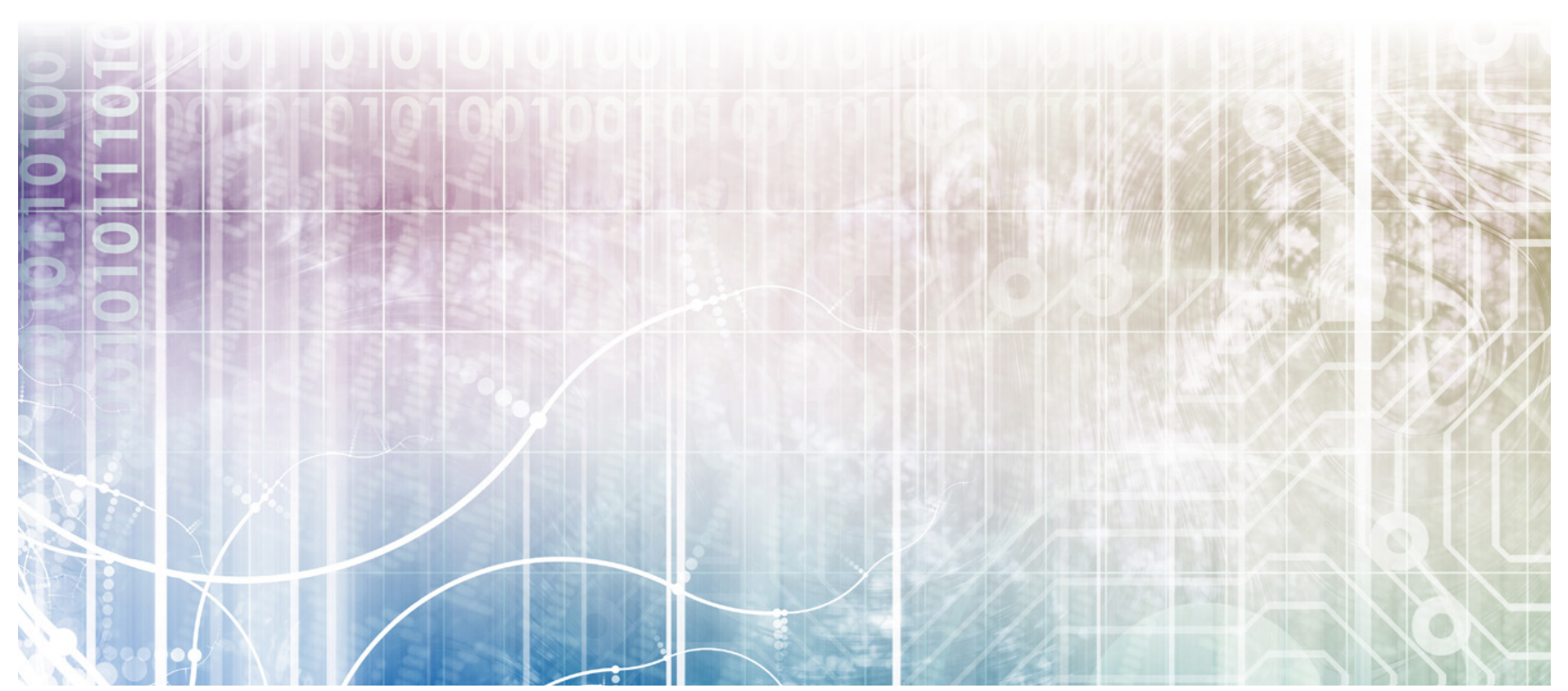

